\title{
Fertility-preserving treatment in women with early endometrial cancer: the Chinese experience
}

This article was published in the following Dove Press journal:

Cancer Management and Research

\section{Yao Wang \\ Jia-xin Yang}

Department of Obstetrics and Gynecology, Peking Union Medical College Hospital, Chinese Academy of Medical Sciences and Peking Union Medical College, Beijing 100730, China
Correspondence: Jia-xin Yang Department of Obstetrics and Gynecology, Peking Union Medical College Hospital, Chinese Academy of Medical Sciences and Peking Union Medical College, No. I Shuaifuyuan, Dongcheng District, Beijing 100730, China

Tel +861069154022

Fax +86 I0 6915 5635

Email yangjiaxin_2017@I63.com

\begin{abstract}
Endometrial cancer (EC) is the most common malignant tumor of the female reproductive tract in developed countries and the second most common gynecologic malignancy in China. Although EC is most often diagnosed in postmenopausal women, the increase in obesity rates and other lifestyle changes has been accompanied by an increase in the prevalence of EC among younger women. Approximately $4 \%$ of patients are diagnosed before the age of 40 years and before having given birth. Such patients may have a strong desire to preserve their fertility despite oncologic risks and thus may be reluctant to undergo standard treatment for EC, which includes hysterectomy, bilateral salpingo-oophorectomy, and surgical staging. Therefore, more conservative treatment approaches may need to be discussed, along with their associated oncologic and reproductive outcomes. In fact, various studies have confirmed the effectiveness and safety of conservative treatment in EC. This review aimed to provide a concise yet informative overview of the current status of fertility-preserving treatment for EC in China, as well as to summarize the latest and ongoing research efforts in this area.
\end{abstract}

Keywords: endometrial cancer, conservative treatment, Chinese, fertility

\section{Introduction}

Endometrial cancer (EC) is the leading gynecologic malignancy in developed countries. In recent years, with the increase in global obesity rates, the incidence rate of EC in China has also risen, with $\sim 63,400$ newly diagnosed cases in $2015 .{ }^{1}$ While EC is more prevalent in postmenopausal women, $7.1 \%$ of patients are diagnosed between the ages of 20 and 44 years. $^{2}$ As a substantial number of reproductive-age women are delaying childbearing, the rate of EC diagnosis in nulliparous women has been increasing as well. In fact, it is estimated that $70 \%-88 \%$ of women with EC in this population have not completed childbearing at the time of diagnosis. ${ }^{3}$ In addition, since the adoption of the two-child per family policy in China, an increasing number of EC patients have a strong desire to preserve their fertility. However, the standard management for EC involves hysterectomy and bilateral salpingo-oophorectomy with or without pelvic lymphadenectomy, which deprives patients of the chance of future pregnancy. Conservative treatment has emerged as an important alternative for younger EC patients who desire to retain their fertility despite oncologic risks. Therefore, the effectiveness of conservative treatment for EC represents an important topic of discussion.

In younger women with EC, conservative treatment is feasible because many ECs diagnosed in this population are early-stage, well-differentiated endometrioid endometrial carcinomas with favorable prognosis. ${ }^{2}$ Indeed, several studies have confirmed 
the effectiveness of conservative treatment for $\mathrm{EC}, 4,5$ reporting that younger patients with low-grade EC had excellent survival regardless of the primary therapy chosen (hormone therapy vs primary surgery). ${ }^{6}$

In China, the conservative treatment of gynecological malignancies is based on the "Standardization study on the preservation of reproductive function in gynecological malignant tumors", a key project of the "Eleventh Five-Year Plan" (2006-2010), supported by the National Science and Technology Foundation. Since then, major clinical centers in China have begun providing fertility-preserving treatments to women with early-stage cervical, endometrial, or ovarian cancers. However, the effectiveness of such treatments varies substantially in clinical practice, and clinicians often find it challenging to address certain issues. In 2014, the Gynecologic Oncology Branch of the Chinese Medical Association established the clinical diagnostic and therapeutic guidelines for fertility-preserving treatment of gynecological malignancies, in order to provide patients and medical practitioners with up-to-date scientific evidence and recommendations for effective therapeutic strategies. ${ }^{7}$ Since then, the conservative treatment of gynecological malignancies has received increasing attention, and many related studies are currently underway. The present review summarizes the current state of fertility-preserving treatment for EC in China and provides a literature overview reflecting the latest research trends in this direction.

\section{Candidates for conservative treatment}

The guidelines developed by the Gynecologic Oncology Branch of the Chinese Medical Association establish the eligibility of early-stage EC patients for fertility-preserving treatment based on the following: ${ }^{7} 1$ ) age $<40$ years; 2) strong desire to preserve fertility; 3 ) diagnosis of well-differentiated endometrioid adenocarcinoma; 4) lesions confined to the endometrium, without myometrial infiltration or extrauterine metastasis; 5) positive progesterone receptor (PR) status for patients who are candidates for progestin therapy; 6) no contraindications to progestin for patients who are candidates for progestin therapy; 7) good compliance and full understanding of the advantages and risks associated with conservative treatment.

Currently, domestic and international guidelines agree that only patients with highly differentiated endometrioid adenocarcinoma, strong fertility desire, and no myometrial invasion may be considered for conservative therapy, ${ }^{7-10}$ because such patients have a low risk of cancer progression during conservative treatment. In fact, even if disease progression occurs, such patients have a 5-year survival rate comparable to that of women who received definitive surgery at diagnosis. ${ }^{10}$ New research indicates that well-differentiated G1 endometrioid adenocarcinoma with minimal myometrial invasion can be treated with conservative hysteroscopic surgery. ${ }^{11}$ However, due to the limited number of cases studied to date, the safety and efficacy of conservative treatment in this particular population remain to be confirmed. In addition, only the Chinese guideline maintains the age requirement (aged $\leq 40$ years) for EC patients to be considered candidates for conservative treatment. With the adoption of China's second-child policy and the development of assisted reproductive technology (ART), it remains to be determined whether the age requirement for fertility-preserving treatment for EC may be relaxed.

\section{Pretreatment assessment}

Preretreatment assessment is particularly important and takes into consideration disease history, previous treatment methods and associated response, comorbidities, checkup findings, physical status, pathological findings, and lesion severity based on imaging examination or laparoscopy. ${ }^{7}$ While the optimal imaging modality for pretreatment assessment of myometrial invasion has not been established, transvaginal ultrasound and magnetic resonance imaging are frequently used. ${ }^{10}$ Imaging is not only helpful to detect myometrial invasion but also to exclude synchronous ovarian tumors or suspicious lymphadenopathy. Although laparoscopic evaluation of metastatic status is not currently recommended, laparoscopy and biopsy are necessary under certain special circumstances such as in patients with imaging-suspected concurrent ovarian cancer or lymph node involvement.

\section{Conservative treatment options Hormonal therapy}

Progestin treatment is the most frequently reported approach for conservative management of early-stage EC. In 1997, Kim et al first reported that EC patients conceived successfully after progestin treatment. ${ }^{12}$ Since then, a number of retrospective studies have demonstrated the role of progestin therapy in the conservative management of EC. ${ }^{13}$ A total of 15 studies covering 405 patients with stage IA EC treated with progestin were published between 2006 and 2017 in China (Table 1). ${ }^{4,-27}$ In China, the therapeutic effect of progestin agents was first described in 2006 by Yu et al, ${ }^{27}$ who described 25 patients ( 8 with EC and 17 with severe atypical hyperplasia) treated with medroxyprogesterone acetate 


\begin{tabular}{|c|c|c|c|c|c|c|c|c|c|c|c|c|c|c|c|c|c|c|}
\hline 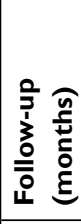 & & $\begin{array}{l}\frac{5}{T} \\
1 \\
n\end{array}$ & $\begin{array}{l}a \\
\bar{a} \\
\frac{a}{m}\end{array}$ & 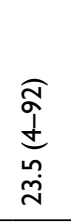 & 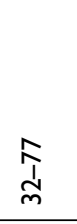 & 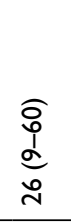 & $\S$ & 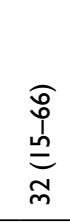 & 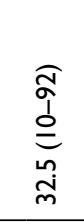 & 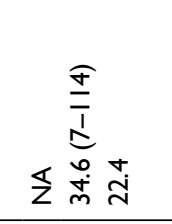 & 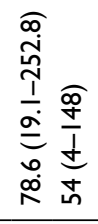 & సై & $\begin{array}{l}5 \\
\vdots \\
y \\
y \\
y\end{array}$ & $\frac{2}{\frac{1}{2}}$ & & Z & $\begin{array}{l}E \\
i \\
E \\
\sigma \\
j\end{array}$ & $\begin{array}{l}\bar{a} \\
\text { d } \\
\end{array}$ \\
\hline 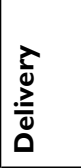 & & 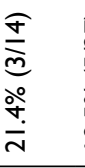 & 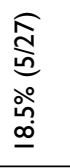 & $\begin{array}{l}\frac{\widehat{o}}{c} \\
\stackrel{0}{\circ} \\
\stackrel{m}{0}\end{array}$ & 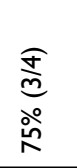 & 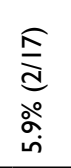 & 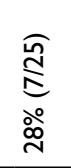 & 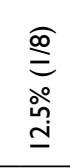 & $\begin{array}{l}\widehat{\bar{స}} \\
\sqrt[\omega]{0} \\
\stackrel{\circ}{0} \\
\stackrel{\sim}{j}\end{array}$ & 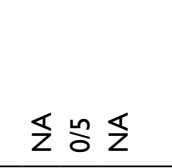 & 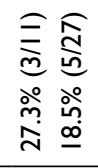 & 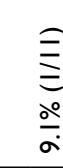 & 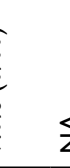 & ¿ & & 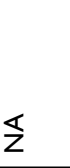 & $\begin{array}{l}\stackrel{\kappa}{c} \\
\stackrel{0}{\circ} \\
\stackrel{+}{+}\end{array}$ & $\begin{array}{l}\widehat{o} \\
\frac{0}{0} \\
\text { ○ें } \\
\text { ì }\end{array}$ \\
\hline 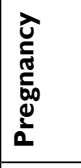 & & 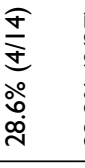 & 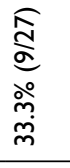 & 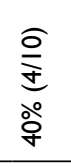 & $\begin{array}{l}\frac{\pi}{m} \\
\stackrel{0}{\circ} \\
\stackrel{\circ}{i}\end{array}$ & 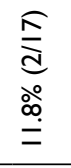 & 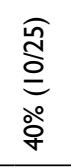 & 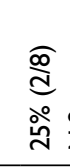 & 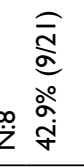 & 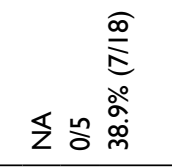 & 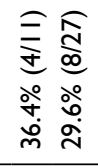 & 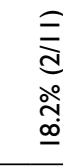 & 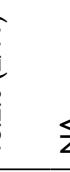 & ¿ & & z & $\begin{array}{l}\frac{F}{y} \\
\stackrel{0}{7} \\
\text { in }\end{array}$ & 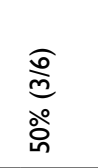 \\
\hline 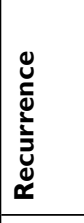 & & 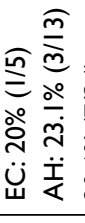 & $\begin{array}{l}\overparen{5} \\
\stackrel{0}{5} \\
\stackrel{0}{0} \\
\stackrel{0}{0}\end{array}$ & $\begin{array}{l}\stackrel{\frac{n}{2}}{c} \\
\stackrel{\text { ’े }}{ }\end{array}$ & ¿ & 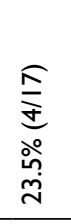 & $\S$ & 号 & 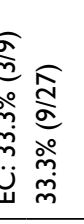 & 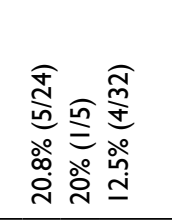 & 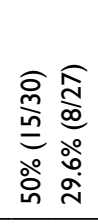 & 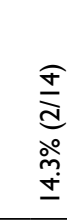 & 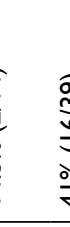 & 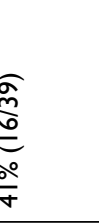 & & 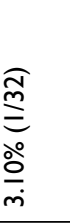 & $\begin{array}{l}\stackrel{E}{\Xi} \\
\stackrel{\circ}{m} \\
\stackrel{ \pm}{ \pm} \\
\end{array}$ & 0 \\
\hline $\begin{array}{l}\text { Uूँ } \\
\text { Ũ } \\
\end{array}$ & & 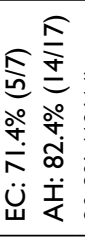 & 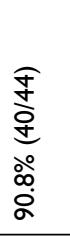 & 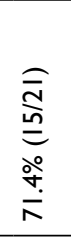 & $\begin{array}{l}\widehat{o} \\
\stackrel{y}{0} \\
\stackrel{0}{\hat{0}} \\
0\end{array}$ & 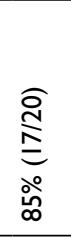 & 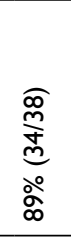 & $\begin{array}{l} \\
\circ \\
\infty \\
\infty \\
\infty\end{array}$ & 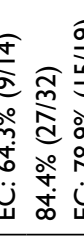 & 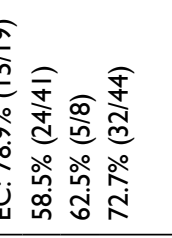 & 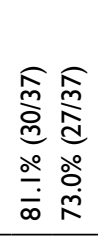 & 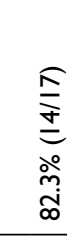 & סִ & 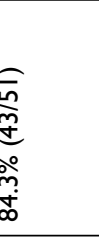 & & 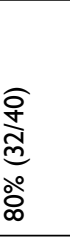 & 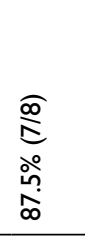 & $\begin{array}{l}\widehat{0} \\
\stackrel{0}{0} \\
\stackrel{0}{0} \\
0\end{array}$ \\
\hline 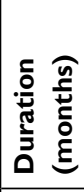 & & 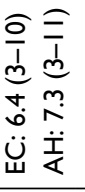 & & $\begin{array}{c}\widehat{T} \\
\frac{1}{0} \\
0\end{array}$ & 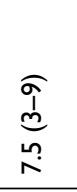 & $\begin{array}{l}\frac{\widehat{\omega}}{1} \\
\stackrel{1}{\leftrightarrows}\end{array}$ & 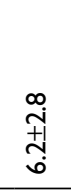 & 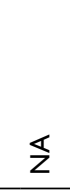 & กี & 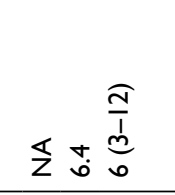 & 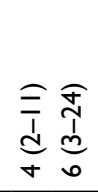 & $\begin{array}{l}\frac{\bar{m}}{+} \\
\frac{++}{\sigma}\end{array}$ & & $\frac{\substack{m \\
\frac{m}{1} \\
\frac{m}{b}}}{b}$ & & ¿ & $\stackrel{a}{m}$ & $\S$ \\
\hline 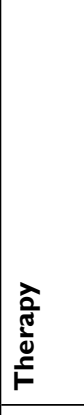 & & 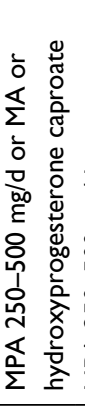 & 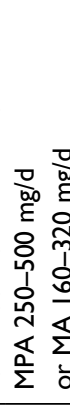 & 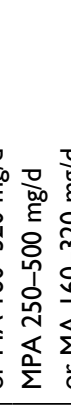 & 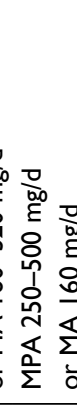 & 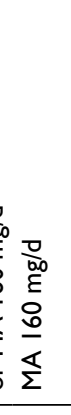 & 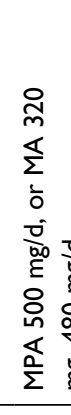 & 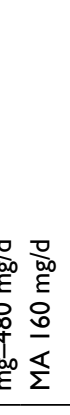 & 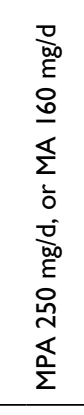 & 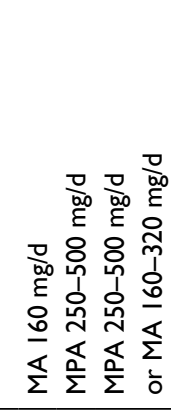 & 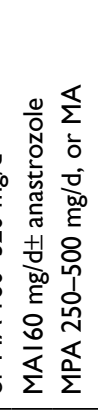 & 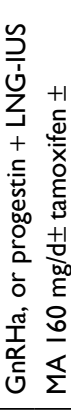 & 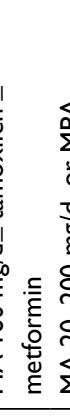 & 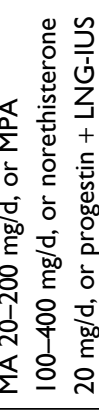 & 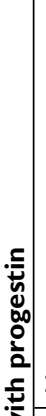 & 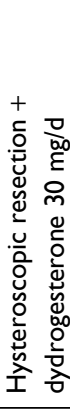 & 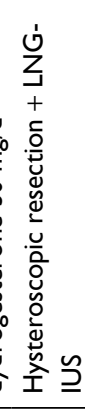 & 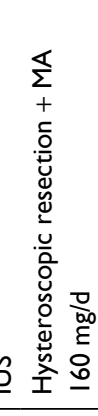 \\
\hline 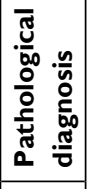 & & 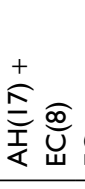 & & u & u & $\begin{array}{l}+ \\
\frac{+}{6} \\
\frac{1}{4} \\
5\end{array}$ & & 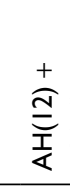 & 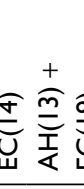 & Eu & u & u & 4 & u & & u & u & u \\
\hline z & $\stackrel{4}{2}$ & $\stackrel{\sim}{\sim}$ & $\stackrel{\infty}{+}$ & $\grave{\sim}$ & 0 & 요 & $\stackrel{\infty}{m}$ & $\stackrel{2}{\sim}$ & $\tilde{m}$ & $\bar{\sigma} \infty \quad \overline{7}$ & $\hat{m} \hat{m}$ & & $\bar{J}$ & $\bar{n}$ & & 우 & $\infty$ & 0 \\
\hline 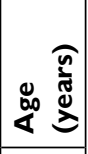 & 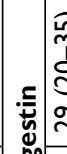 & 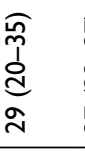 & 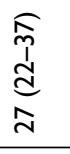 & $\begin{array}{l}\frac{\sigma}{d} \\
\frac{1}{d} \\
\frac{m}{m}\end{array}$ & $\begin{array}{l}\text { ô. } \\
\stackrel{d}{d} \\
\infty \\
\text { d }\end{array}$ & 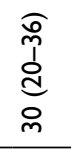 & 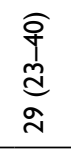 & $\begin{array}{l}\text { } \\
\stackrel{\infty}{0} \\
\stackrel{\infty}{=} \\
0 \\
0\end{array}$ & 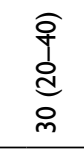 & 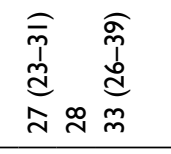 & 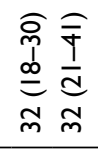 & $\bar{m}$ & 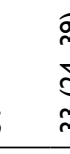 & 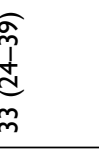 & & 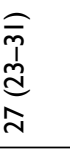 & $\S$ & $\begin{array}{l}\text { } \\
i \\
\stackrel{d}{d} \\
0 \\
0\end{array}$ \\
\hline 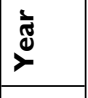 & 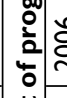 & ઠัڤ & กั & $\overline{\bar{i}}$ & 음 & $\stackrel{\sim}{\alpha}$ & $\stackrel{m}{i}$ & $\frac{m}{i}$ & $\stackrel{n}{\grave{i}}$ & 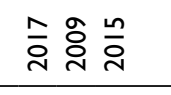 & 훙 & $\overline{\bar{i}}$ & $\underline{c}$ & $\stackrel{n}{i}$ & & ì & $\frac{m}{i}$ & $\stackrel{n}{\circ}$ \\
\hline $\begin{array}{l}\text { ते } \\
\text { जै }\end{array}$ & 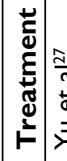 & 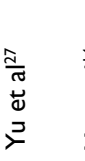 & 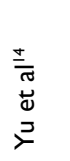 & 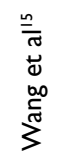 & 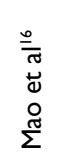 & 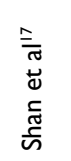 & $\begin{array}{l}\frac{\infty}{\pi} \\
\tilde{J} \\
0 \\
\tilde{U}\end{array}$ & $\begin{array}{l}\frac{a}{\bar{d}} \\
\Delta \\
\frac{0}{\sigma} \\
\frac{\sigma}{\omega}\end{array}$ & $\begin{array}{l}\frac{0}{\pi} \\
\stackrel{0}{0} \\
\frac{0}{\sigma} \\
\frac{N}{N}\end{array}$ & 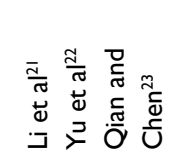 & 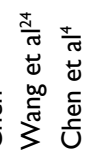 & 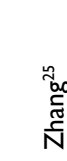 & $\begin{array}{ll}2 \\
\vdots \\
0 \\
\vdots \\
\vdots\end{array}$ & 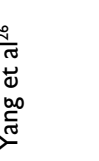 & & 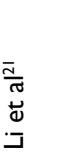 & $\begin{array}{l}\frac{m}{\omega} \\
\stackrel{m}{0} \\
ت \\
ت\end{array}$ & 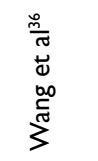 \\
\hline
\end{tabular}




\begin{tabular}{|c|c|c|c|c|c|c|c|}
\hline 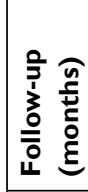 & 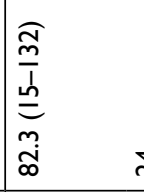 & \pm & 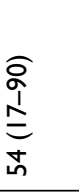 & ¿ & ¿ & & 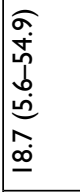 \\
\hline 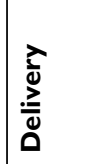 & 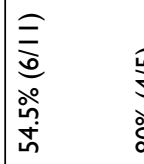 & 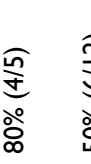 & 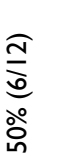 & 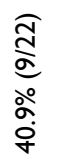 & 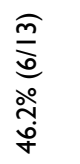 & & $\frac{m}{\delta}$ \\
\hline 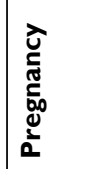 & 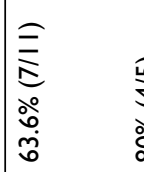 & 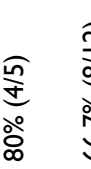 & 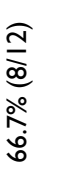 & 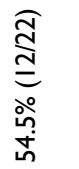 & 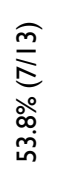 & & 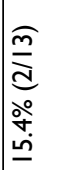 \\
\hline 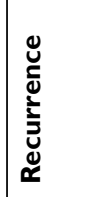 & 0 & 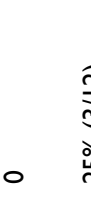 & 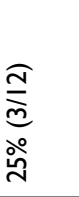 & 0 & 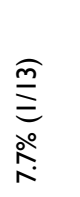 & & 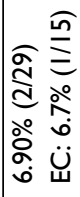 \\
\hline 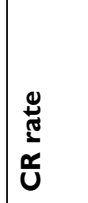 & 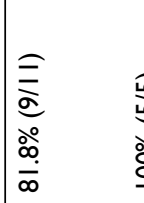 & 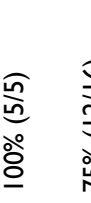 & 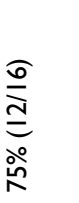 & 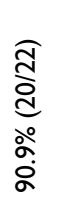 & 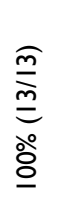 & & 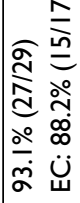 \\
\hline 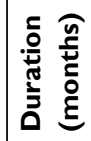 & 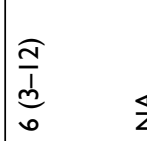 & 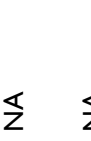 & $§$ & 으 & 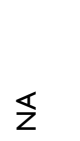 & & $\begin{array}{l}0 \\
\text { i } \\
+1 \\
0 \\
\text { in }\end{array}$ \\
\hline $\begin{array}{l}\frac{\partial}{\frac{\partial}{d}} \\
\frac{E}{E}\end{array}$ & 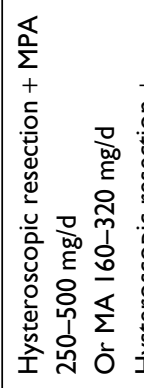 & 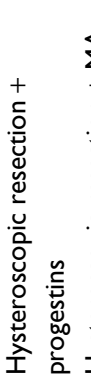 & 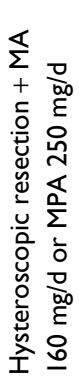 & 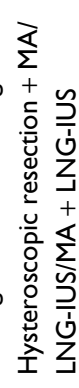 & 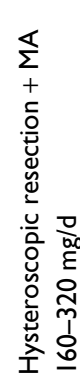 & $\begin{array}{l} \\
\\
0 \\
0 \\
0 \\
0 \\
0 \\
0 \\
0\end{array}$ & 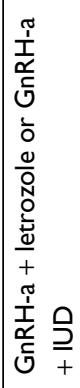 \\
\hline 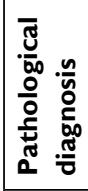 & u & U & u & u & $\underset{\Psi}{u}$ & 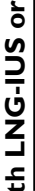 & 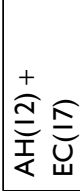 \\
\hline$\stackrel{\circ}{\mathbf{z}}$ & $=$ & n & 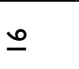 & ה & $\underline{m}$ & $\begin{array}{l}\overline{3} \\
\bar{d} \\
\end{array}$ & নি \\
\hline 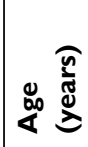 & 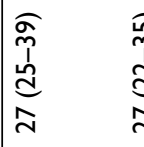 & 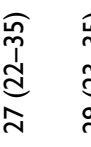 & 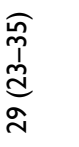 & $\begin{array}{l}\underset{\mathfrak{p}}{1} \\
\stackrel{1}{d} \\
m \\
m\end{array}$ & 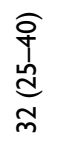 & 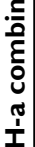 & $\frac{\widehat{T}}{\frac{y}{d}}$ \\
\hline 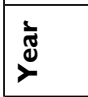 & $\bar{i}$ & 음 & 슷 & $\frac{0}{\circ}$ & $\frac{\text { 늠 }}{\grave{2}}$ & $\begin{array}{l}\vec{x} \\
\mathbf{v} \\
\mathbf{v} \\
\underline{0}\end{array}$ & 송 \\
\hline 훌 & 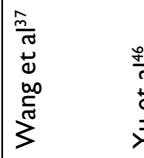 & $\begin{array}{l}j \\
5 \\
\frac{\pi}{2} \\
\frac{\pi}{2} \\
5\end{array}$ & 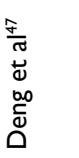 & 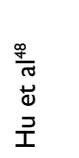 & 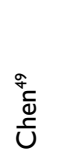 & 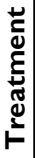 & 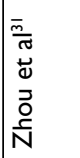 \\
\hline
\end{tabular}

(MPA). The average age of the patients was 28.6 years (range, 20-35 years). Of the eight patients with EC, six responded to treatment (average response time, 2.5 months; range, 1-3 months) and five achieved complete response (CR; average time to CR, 6.4 months; range, 3-10 months). Of those who achieved $\mathrm{CR}$, one $(20 \%)$ relapsed at 30 months after remission. Subsequently, Yu et a ${ }^{14}$ reported the result of a multicenter study examining the role of hormonal therapy in the conservative management of EC. The study period spanned from 1998 to 2011, totaling 48 patients treated with progestins. The median age was 27 years (range, 22-37 years) and $90.8 \%$ (40/48) of patients achieved CR. The average time to CR was $5.3 \pm 2.8$ months. Recurrence was noted in $20.6 \%$ of the 34 patients who had responded to treatment initially and been followed up. The average time to recurrence was 23.7 months (range, $8-45$ months). Later studies reported a CR rate of $58.5 \%-89 \%$, with a recurrence rate of $14.3 \%-50 \%$.

Most patients described in the literature received treatment with either MPA or megestrol acetate (MA), but there are controversies about the efficacy of these progestogens. Whereas MA treatment was previously reported to be associated with higher remission rate and lower recurrence rate, ${ }^{28}$ the latest large-scale clinical study reported comparable efficacy of these two oral progestins, with a lower recurrence rate for MPA. ${ }^{29}$ Therefore, it is worthwhile to continue exploring which medication can yield superior clinical effectiveness. High-dose progesterone therapy is beneficial for reversing EC but causes various adverse side effects. No definitive consensus has been reached on the optimal dose and duration of progestin treatment, which vary across studies. Currently, the most commonly reported regimens include MPA at 250$600 \mathrm{mg} /$ day or MA at $160-480 \mathrm{mg} /$ day. $^{8,30}$ In China, MPA is commonly administered at $250-600 \mathrm{mg}$ /day and MA at $160-320 \mathrm{mg} /$ day. ${ }^{4,14-25}$ Only Yang et $\mathrm{al}^{26}$ reported the usage of oral progestogens at lower doses, with MPA at $100-400 \mathrm{mg} /$ day and MA at 20-200 mg/day without interruption.

\section{Hysteroscopic resection and progesterone therapy}

Long-term oral administration of large doses of progesterone has adverse side effects, which restricts its use in certain populations and limits compliance among young women. Therefore, research efforts have focused on developing a more effective and convenient treatment to help improve compliance and alleviate the side effects associated with oral progesterone. Other strategies including levonorgestrel-releasing intrauterine system (LNG-IUS) and gonadotropin-releasing 
hormone agonist (GnRH-a) therapy, used alone or in combination, have been explored as alternative options for conservative therapy in women with EC because the effectiveness of such strategies is comparable to that of high-dose oral progesterone. . $^{71,32}$

Hysteroscopic resection combined with progesterone therapy is a new type of conservative treatment. Hysteroscopic resection involves resection of the tumor, of a small layer of the myometrium underlying the lesion (two-step technique), and of the endometrium adjacent to the tumor (three-step technique). ${ }^{33}$ More than a decade of research has not only confirmed that hysteroscopic resection is effective for treating early-stage, highly differentiated endometrioid adenocarcinoma in patients with a strong desire to retain fertility, but that hysteroscopic resection is superior to traditional conservative treatment. ${ }^{33}$ The findings of the two largest studies on the effect of fertility-sparing, combined therapy involving hysteroscopic resection and medical treatment strongly suggest that this combined approach is comparable to progestin therapies in terms of treatment response rates and pregnancy rates. ${ }^{33,34}$ In 2013 , Li et $\mathrm{al}^{35}$ described the first Chinese case series involving patients who received combined hysteroscopic resection and progestin therapy. This case series included eight patients who underwent hysteroscopic resection of the endometrial lesions and shallow muscular layer within 2-3 $\mathrm{mm}$ below the lesion, followed by insertion of an LNG-IUS (Mirena; Bayer HealthCare Pharmaceutical Inc, Whippany, NJ, USA). Seven of eight patients achieved $\mathrm{CR}$, whereas one patient had tumor relapse and underwent hysterectomy and bilateral salpingo-oophorectomy at 5 months after hysteroscopic resection. Four patients became pregnant within 3-6 months after treatment completion, and three patients delivered successfully. Later, Wang et $\mathrm{al}^{36}$ reported a series of six patients who underwent hysteroscopic resection of the local lesion, of the shallow muscular layer, and of the surrounding tissues within at least $5 \mathrm{~mm}$ (three-step technique). In all patients, EC was confirmed as stage IA, and MA treatment was indicated at $160 \mathrm{mg} /$ day for 20 days each month or continuously for 3 months. All six patients achieved $\mathrm{CR}$ at 3 months and no recurrence occurred during an average follow-up of 48.5 months. Three patients conceived naturally and delivered healthy infants at full term. A similar study conducted by Wang et $\mathrm{al}^{37}$ described 11 young women with stage IA EC, who received hysteroscopic resection (twostep technique) combined with progestin therapy (MPA at $250-500 \mathrm{mg}$ /day or MA at $160-320 \mathrm{mg}$ /day). All patients achieved at least partial remission within 3 months, and nine achieved CR within 3-12 months. During an average follow- up of 82.3 months, two patients underwent definitive surgery after completion of fertility and all patients were disease-free. Such findings were consistent between studies conducted in China and those conducted in other countries, suggesting that combined treatment involving hysteroscopic endometrial focal resection followed by LNG-IUS insertion can be an effective and safe approach for the management of EC in young women who desire to preserve fertility. Nevertheless, any recommendations based on these conclusions should be drafted with utmost care because the supporting studies had retrospective design and covered a limited number of patients.

\section{GnRH-a therapy combined with LNG- IUS insertion or oral letrozole}

Previous studies have confirmed the effectiveness of GnRH-a in patients with EC. ${ }^{38,39}$ In recent years, LNG-IUS insertion has been proposed as an alternative option for treating endometrial intraepithelial neoplasia and EC. ${ }^{40}$ Unlike oral progestin, LNG-IUS provides very high doses of progestin to the local endometrium without serious systemic adverse effects. Letrozole, which is a third-generation aromatase inhibitor, was reported to have synergistic effect with the above mentioned therapies for reversing EC. ${ }^{41,42}$ In China, only one study covered cases of EC and complex atypical hyperplasia $(n=29)$ managed conservatively with combined GnRH-a and LNG-IUS/letrozole therapy. ${ }^{31}$ During a median follow-up of 18.7 months, 88.2\% (15/17) of patients with EC achieved CR, one of whom had recurrence.

\section{Management after completion of conservative treatment Women with a desire to conceive}

Patients who have achieved CR and wish to conceive are advised to attempt to do so as soon as possible, especially if they have risk factors such as thin endometrium, older age, and history of recurrence. ${ }^{43}$ Pregnancy can reduce the rate of recurrence, ${ }^{44}$ and it is generally believed that it is safe for patients to become pregnant starting at 3 months after achieving CR. ${ }^{45}$ However, regular oncologic follow-up should be continued during pregnancy, maintaining a follow-up interval of 6 months. ${ }^{10}$

The published literature confirms that patients with earlystage EC managed conservatively have promising fertility outcomes. The pregnancy data from studies conducted in China are summarized in Table 1. The only multicenter study, which was conducted by Yu et al, ${ }^{14}$ reported that $33.3 \%$ $(9 / 27)$ of the treated women became pregnant and $18.5 \%$ 
(5/27) delivered successfully after completing therapy. The average time from treatment completion to pregnancy was 12.2 months (range, 4-25 months). Subsequent studies that included patients aged $>15$ years reported pregnancy rates ranging from $12.5 \%$ to $50 \%$ and delivery rates ranging from $9.1 \%$ to $30 \%$ after progestin therapy. ${ }^{14,15,18,20,21,23,24,26}$ As for the pregnancy outcome of patients managed by the hysteroscopic approach, seven studies provided pregnancy data, reporting pregnancy rates ranging from $50 \%$ to $80 \%$ and live-birth rates ranging from $40.9 \%$ to $80 \%{ }^{35-37,46-49}$ However, it should be noted that the highest documented pregnancy and birth rates were based on a series of only five patients. ${ }^{46}$ Although conventional wisdom suggests that hysteroscopic resection may lead to complications such as intrauterine adhesions and intimal fibrosis, which may affect pregnancy, no intrauterine adhesions were noted in previous studies on hysteroscopic resection, which reported very high pregnancy and live-birth rates. These findings suggest that hysteroscopic resection may have no significant adverse effects on pregnancy outcomes, though further verification is warranted. The only study of patients receiving GnRH-a combined with letrozole/LNGIUS therapy, two of 13 women who attempted to conceive achieved pregnancy spontaneously, with no successful delivery. Twelve women who attempted to conceive underwent ART but there was no delivery until the last follow-up. ${ }^{31}$

Regarding the optimal choice of reproductive strategy following conservative treatment, EC patients with no history of infertility may consider natural conception, but the pregnancy rate is expected to be low due to thin endometrium. Therefore, ARTs are recommended in women with a strong desire to conceive following EC treatment. Indeed, several studies have shown that pregnancy rate is higher in patients who used ART than in those who attempted natural conception $^{20,20,50,51}$ and that ART does not affect the prognosis of EC. ${ }^{6,52}$ However, an increased incidence of perinatal complications including preterm birth, preeclampsia, prolificacy, and need for cesarean section was reported by Chao et $\mathrm{a}^{53}$ in EC patients who became pregnant using ART. Therefore, women attempting to conceive using ART should undergo close antenatal follow-up.

\section{Women without pregnancy intention}

For patients who have achieved CR but do not intend to become pregnant, the clinical practice guidelines issued by the European Society for Medical Oncology recommend oral administration of low-dose progesterone but do not establish a specific regimen (dosage, continuous or periodic administration). ${ }^{10,54}$ The Chinese guideline suggests that both periodic treatment with oral short-acting contraceptives and LNG-IUS insertion represent suitable options.

\section{Women who have completed childbearing}

Because recurrence rates after $\mathrm{CR}$ remain high, almost all clinical practice guidelines recommend that patients who have completed fertility should undergo hysterectomy. ${ }^{10,54}$ The Chinese guideline also recommends that patients who have completed fertility can be inserted with an LNG-IUS to prevent tumor recurrence. ${ }^{7}$ As an increasing number of patients plan to conceive more than one child, further study should clarify whether patients who have completed fertility can receive maintenance therapy only, allowing the possibility of future pregnancy.

\section{Women with cancer recurrence}

$\mathrm{EC}$ recurrence is defined as the presence of an endometrial lesion after CR to conservative treatment. EC recurrence rates following $\mathrm{CR}$ remain high $(24 \%-40 \%) .{ }^{28,30,55} \mathrm{To}$ develop strategies for decreasing the risk of EC recurrence, it is necessary to identify the influencing factors. Koskas et $\mathrm{al}^{28}$ conducted a systematic review of prognostic factors of oncologic and reproductive outcomes in fertility-sparing management of EC. This study included 370 patients from 24 studies and reported that none of the factors evaluated (age, gravidity status, obesity, previous infertility, hormonal therapy) was associated with an increased risk of recurrence. However, in China, a retrospective study conducted by Chen et $\mathrm{al}^{4}$ reported that older age ( $\geq 35$ years), obesity (body mass index $\geq 30 \mathrm{~kg} / \mathrm{m}^{2}$ ), longer time to $\mathrm{CR}$, and consistent infertility after conservative treatment were associated with an increased risk of recurrence. Whether factors mentioned above are the influencing factors of recurrence deserves future investigation.

There is no consensus regarding the optimal management strategy for patients with early-stage EC who experience recurrence after fertility-preserving treatment. Only the guidelines issued in 2015 by the European Society for Gynecological Oncology indicate that high-dose progestogen therapy can be given again to patients who relapse after $\mathrm{CR}$, but this recommendation is restricted to nulliparous women. ${ }^{10}$ Some researchers suggest that standard surgery including hysterectomy should be performed immediately in patients with recurrence, while others propose that repeated conservative treatment is safe and effective in patients with recurrence who still meet the criteria for initial conservative treatment. ${ }^{10} \mathrm{~A}$ limited number of studies reported that 
patients who relapsed after fertility-preserving therapy achieved $\mathrm{CR}$ again with prolonged progesterone therapy (Table 2). ${ }^{56-62}$ In China, the largest cohort study focused on repeated conservative treatment in patients with recurrent EC was conducted by $\mathrm{Yu}$ et $\mathrm{a}^{63}$ in Peking Union Medical College Hospital. Of the 36 patients with recurrence after primary fertility-sparing treatment, $76.2 \%$ achieved CR again after a second round of conservative treatment. Two of 16 patients who achieved $\mathrm{CR}$ and attempted to conceive became pregnant and delivered successfully. These results suggest promising outcomes of repeated conservative treatment in patients with recurrent EC after CR to primary fertility-preserving treatment. Future clinical studies should clarify the optimal regimen (dose, timing) and follow-up protocol (timing, investigations) for repeated conservative treatment after relapse.

\section{Other factors affecting the indication for conservative treatment}

The risk of hereditary nonpolyposis colorectal cancer (Lynch/ HNPCC) syndrome in EC patients younger than 50 years is $5 \%-10 \%{ }^{64}$ Therefore, patients with early-stage EC and a family history of colorectal cancer should undergo immunohistochemistry evaluation to determine the expression of Lynch syndrome-related genes (MLH1, MSH2, MSH6, and PMS2) before deciding the indication for conservative treatment. Patients with loss of expression of these genes should undergo a gene test; if genetic defects are found, conservative treatment should not be indicated or should be stopped. At present, there is no effective treatment for EC in patients with Lynch syndrome. Future advances in conservative treatment strategies may allow patients with Lynch syndrome to become suitable candidates for conservative treatment, but only under close follow-up.

\section{Progress in basic research}

Progestin resistance is the main obstacle to successful conservative therapy in young women with EC, as up to $30 \%$ of patients fail to respond to progestin due to primary or acquired progestin resistance. ${ }^{65,66}$ Several studies have investigated the mechanisms contributing to progestin resistance. Survivin, which is a key regulator of antiapoptotic networks, is overexpressed in both endometrial hyperplasia and EC. ${ }^{67,68}$ Chen et al ${ }^{69}$ found that the expression of survivin can be downregulated by progestin. Dysregulation of survivin expression may contribute to progestin resistance in the hyperplastic endometrium. Progesterone inhibits the proliferation of EC cells and reverses their malignant biological behavior through binding to the nuclear PR. Thus, progestin cannot inhibit EC cells that do not express PR and may even promote cell proliferation. ${ }^{70} \mathrm{Gu}$ et $\mathrm{al}^{70}$ showed that, in the absence of PR mediation, progestin-induced activation of the PI3K/Akt pathway plays an important role in progestin resistance of EC cells, suggesting that inhibition of the PI3K/ Akt pathway might restore PR expression and reverse progestin resistance in EC. Other pathways involved in progestin resistance include upregulation of EGFR expression, overexpression of glyoxalase I, and insulin resistance. ${ }^{71-73} \mathrm{Ai}$ et al ${ }^{73}$ indicated that overexpression of EGFR reduced the sensitivity of EC cells to progestin and inhibited cell apoptosis. Thus, EGFR tyrosine kinase inhibitors can suppress the proliferation of EC cells and may be effective in the treatment of EC. Furthermore, Zhang et $\mathrm{al}^{72}$ demonstrated that metformin can reverse progestin resistance by downregulation of glyoxalase I expression, suggesting that combined therapy with metformin and progestin may be effective in treating EC.

\section{Ongoing clinical trials}

Nowadays, several prospective clinical trials aiming to evaluate different strategies for conservative management of

Table 2 Studies on repeated treatment of recurrent stage I EC

\begin{tabular}{|c|c|c|c|c|c|c|c|}
\hline Study & Year & No. of cases & Primary CR & Recurrence & $\begin{array}{l}\text { No. of repeated } \\
\text { treatment }\end{array}$ & Second CR & $\begin{array}{l}\text { Follow-up time } \\
\text { (months) }\end{array}$ \\
\hline Gotlieb et $\left.a\right|^{56}$ & 2003 & $13 \mathrm{EC}$ & $100 \%$ & $46.2 \%(6 / 13)$ & 4 & $100 \%(4 / 4)$ & $6-358$ \\
\hline Ramirez et $\mathrm{a}^{57}$ & 2004 & $81 \mathrm{EC}$ & $76 \%(62 / 81)$ & $24 \%(15 / 62)$ & 7 & $72 \%(5 / 7)$ & $36-90$ \\
\hline \multirow[t]{2}{*}{ Ushijima et $a^{58}$} & 2007 & $28 \mathrm{EC}+17 \mathrm{AH}$ & EC: $55 \%(12 / 22)$ & EC: $57.1 \%(8 / 14)$ & 8 & $75 \%(6 / 8)$ & $25-73$ \\
\hline & & & $\mathrm{AH}: 82 \%(14 / 17)$ & $\mathrm{AH}: 38 \%(6 / 16)$ & & & \\
\hline Perri et al ${ }^{59}$ & 2011 & $27 \mathrm{EC}$ & $89 \%(24 / 27)$ & $62 \%(15 / 24)$ & II & $100 \%(11 / 11)$ & $7.8-412$ \\
\hline Park et $\mathrm{al}^{60}$ & 2013 & NA & NA & $31.9 \%(45 /|4|)$ & 33 & $84.8 \%(28 / 33)$ & $24-160$ \\
\hline Yamagami et $\mathrm{a}^{\mid 61}$ & 2018 & $97 \mathrm{EC}$ & $90.7 \%(88 / 97)$ & $63.2 \%(55 / 87)$ & 53 & $98.1 \%(53 / 54)$ & $4.5-208.7$ \\
\hline Tamauchi et al ${ }^{62}$ & 2018 & $9 \mathrm{EC}$ & $88.9 \%(8 / 9)$ & $87.5 \%(7 / 8)$ & 6 & $100 \%(6 / 6)$ & NA \\
\hline Yu et $\mathrm{al}^{63}$ & 2018 & $156 \mathrm{EC}$ & $93.6 \%(146 / 156)$ & $34.6 \%(36 / 104)$ & 25 & $76.2 \%(16 / 21)$ & 9-77 \\
\hline
\end{tabular}

Abbreviations: $\mathrm{CR}$, complete response; $\mathrm{AH}$, atypical hyperplasia; $\mathrm{EC}$, endometrial cancer; $\mathrm{NA}$, not available. 
Table 3 List of ongoing clinical trials in China

\begin{tabular}{|c|c|c|c|c|c|}
\hline NCT number & Status & Study title & Conditions & Interventions & Locations \\
\hline NCT03538704 & Recruiting & $\begin{array}{l}\text { Effect of fertility-sparing therapy } \\
\text { of early endometrial cancer }\end{array}$ & Endometrial cancer & Drug: metformin & $\begin{array}{l}\text { Peking University } \\
\text { People's Hospital, Beijing, } \\
\text { China }\end{array}$ \\
\hline NCT03463252 & Recruiting & $\begin{array}{l}\text { Value of LNG-IUS as fertility- } \\
\text { preserving treatment of EAH } \\
\text { and EC }\end{array}$ & $\begin{array}{l}\text { Endometrial cancer; } \\
\text { endometrial atypical } \\
\text { hyperplasia }\end{array}$ & $\begin{array}{l}\text { Drug: progesterone } \\
\text { Device: mirena } \\
\text { Drug: GnRH agonist }\end{array}$ & $\begin{array}{l}\text { West China Second } \\
\text { University Hospital, } \\
\text { Chengdu, Sichuan, China }\end{array}$ \\
\hline NCT0324I9I4 & Recruiting & $\begin{array}{l}\text { Megestrol acetate plus LNG- } \\
\text { IUS in young women with early } \\
\text { endometrial cancer }\end{array}$ & $\begin{array}{l}\text { Endometrial neoplasm } \\
\text { malignant stage I }\end{array}$ & $\begin{array}{l}\text { Drug: megestrol acetate } \\
\text { Device: LNG-IUS }\end{array}$ & $\begin{array}{l}\text { Obstetrics and } \\
\text { Gynecology Hospital, } \\
\text { Fudan University, } \\
\text { Shanghai, China }\end{array}$ \\
\hline NCTOI9683I7 & Completed & $\begin{array}{l}\text { Megestrol acetate plus } \\
\text { metformin to megestrol } \\
\text { acetate in patients with } \\
\text { endometrial atypical hyperplasia } \\
\text { or early stage endometrial } \\
\text { adenocarcinoma }\end{array}$ & $\begin{array}{l}\text { Endometrial atypical } \\
\text { hyperplasia } \\
\text { Endometrial } \\
\text { adenocarcinoma }\end{array}$ & $\begin{array}{l}\text { Drug: megestrol acetate } \\
\text { and metformin } \\
\text { Drug: megestrol acetate }\end{array}$ & $\begin{array}{l}\text { Obstetrics and } \\
\text { Gynecology Hospital, } \\
\text { Fudan University } \\
\text { Fudan University Shanghai } \\
\text { Cancer Center } \\
\text { Shanghai Changning } \\
\text { Maternity and Infant } \\
\text { Health Hospital, Shanghai, } \\
\text { China }\end{array}$ \\
\hline
\end{tabular}

Abbreviations: EC, endometrial cancer; LNG-IUS, levonorgestrel-releasing intrauterine system; EAH, endometrial atypical hyperplasia.

EC are taking place across China (Table 3). Recruitment is currently ongoing for a clinical trial at West China Second University Hospital, aiming to analyze the effectiveness of LNG-IUS insertion for fertility-sparing treatment of endometrial atypical hyperplasia and early endometrial carcinoma (clinicaltrials.gov NCT03463252). Patients who are enrolled are randomly allocated to one of three treatment groups (MPA only, MPA + LNG-IUS, LNGIUS only), and the primary outcomes include pathological response, pregnancy rate, and live-birth rate. The study aims to enroll 224 participants. Recruitment is also ongoing in a prospective, multicenter, open-label, randomized, controlled clinical trial sponsored by Peking Union People's Hospital. The study aims to enroll 80 patients with earlystage EC and body mass index $\geq 25 \mathrm{~kg} / \mathrm{m}^{2}$ to evaluate the effect of fertility-sparing therapy with or without metformin (NCT03538704).

Recruitment was recently completed in a multicenter clinical trial sponsored by Xiaojun Chen aiming to explore whether combined therapy with MA and metformin would be more effective than therapy with MA alone in terms of restoring the endometrial tissue to a normal state in patients with endometrial atypical hyperplasia or early-stage EC (NCT01968317). One hundred and fifty patients have been recruited. Fudan University is currently conducting a noninferiority study comparing the effectiveness of combined therapy with MA + LNG-IUS and therapy with MA alone in terms of restoring the endometrial tissue to a normal state in patients with early-stage EC (NCT03241914). The primary outcomes include pathological response rates and response time in each treatment arm, while secondary outcomes include treatment-related adverse events, rate of relapse, rate of pregnancy, and patients' compliance. This study also evaluates whether the use of combined therapy can shorten the treatment duration, which would represent an advantage in terms of cost and comfort to the patients.

\section{Conclusion}

In younger women with stage IA EC, fertility-preserving treatment is feasible and effective. Apart from oral progestin therapy, LNG-IUS insertion, hysteroscopic resection, and GnRH-a therapy, alone or in combination, have also shown therapeutic promise, but further research is warranted to confirm their effectiveness and safety. After treatment completion, women who wish to conceive are encouraged to attempt to do so as soon as possible. ART is recommended especially in women having a history of infertility. For patients with recurrence after fertility-preserving treatment, repeated fertility-preserving treatment can still achieve a promising response, allowing the possibility of future pregnancy. Further studies are needed to clarify the mechanisms underlying resistance and to develop sensitization strategies in patients with progestin resistance. The outcomes of ongoing and future prospective clinical trials are expected to help clarify the optimal treatment strategy for EC in different patient populations. 


\section{Acknowledgment}

This work was supported by the Chinese Academy of Medical Sciences Initiative for Innovative Medicine (CAMS-2017-I2M-1-002).

\section{Disclosure}

The authors report no conflicts of interest in this work.

\section{References}

1. Chen W, Zheng R, Baade PD, et al. Cancer statistics in China, 2015. CA Cancer J Clin. 2016;66(2):115-132.

2. Corzo C, Barrientos Santillan N, Westin SN, Ramirez PT. Updates on conservative management of endometrial cancer. J Minim Invasive Gynecol. 2018;25(2):308-313.

3. Rackow BW, Arici A. Endometrial cancer and fertility. Curr Opin Obstet Gynecol. 2006;18(3):245-252.

4. Chen M, Jin Y, Li Y, Bi Y, Shan Y, Pan L. Oncologic and reproductive outcomes after fertility-sparing management with oral progestin for women with complex endometrial hyperplasia and endometrial cancer. Int J Gynaecol Obstet. 2016;132(1):34-38.

5. Gunderson CC, Fader AN, Carson KA, Bristow RE. Oncologic and reproductive outcomes with progestin therapy in women with endometrial hyperplasia and grade 1 adenocarcinoma: a systematic review. Gynecol Oncol. 2012;125(2):477-482.

6. Greenwald ZR, Huang LN, Wissing MD, Franco EL, Gotlieb WH. Does hormonal therapy for fertility preservation affect the survival of young women with early-stage endometrial cancer? Cancer. 2017;123(9):1545-1554

7. Gynecologic Oncology Branch of the Chinese Medical Association. Guidelines for fertility-preserving treatment of gynecological malignant tumors (Chinese). Chin J Obstet Gynecol. 2014;4:243-248.

8. Colombo N, Creutzberg C, Amant F, et al. ESMO-ESGO-ESTRO Consensus Conference on Endometrial Cancer: diagnosis, treatment and follow-up. Ann Oncol. 2016;27(1):16-41.

9. Ebina Y, Katabuchi H, Mikami M, et al. Japan Society of Gynecologic Oncology guidelines 2013 for the treatment of uterine body neoplasms. Int J Clin Oncol. 2016;21(3):419-434.

10. Rodolakis A, Biliatis I, Morice P, et al. European society of gynecological oncology task force for fertility preservation: clinical recommendations for fertility-sparing management in young endometrial cancer patients. Int J Gynecol Cancer. 2015;25(7):1258-1265.

11. Casadio P, Guasina F, Paradisi R, Leggieri C, Caprara G, Seracchioli R. Fertility-sparing treatment of endometrial cancer with initial infiltration of myometrium by resectoscopic surgery: a pilot study. Oncologist. 2018;23(4):478-480.

12. Kim YB, Holschneider CH, Ghosh K, Nieberg RK, Montz FJ. Progestin alone as primary treatment of endometrial carcinoma in premenopausal women. Report of seven cases and review of the literature. Cancer. 1997;79(2):320-327.

13. Qin Y, Yu Z, Yang J, et al. Oral progestin treatment for early-stage endometrial cancer: a systematic review and meta-analysis. Int J Gynecol Cancer. 2016;26(6):1081-1091.

14. Yu M, Cao DY, Yang JX, Wu YM, Chen YL, Xie X. Multi-center clinical study of conservative treatment with progestin in early endometrial cancer (Chinese). In: Abstracts of the 10th National Obstetrics and Gynecology Conference of the Chinese Medical Association; Xiamen; 2012:46.

15. Wang YX, Pan LY, Huang HF, Shen K, Lang JH. Clinical analysis of conservative treatment with progestin in young women with endometrial carcinoma. Chin J Cancer Prev Treat. 2011;18(7): 541-544. Chinese.

16. Mao Y, Wan X, Chen Y, Lv W, Xie X. Outcomes of conservative therapy for young women with early endometrial adenocarcinoma. Fertil Steril. 2010;93(1):283-285.
17. Shan BE, Ren YL, Sun JM, Xy T. Jiang zx, Ju XZ. A phase II study of fertility-sparing treatment with megestrol acetate for endometrial carcinoma and atypical hyperplasia in young women (Chinese). China Oncol. 2012;22(6):424-429.

18. Cao DY, Yu M, Yang JX, et al. [Pregnant rate and pregnancy-relating factors of patients with early endometrial carcinoma and severe atypical hyperplasia of endometrium after fertility-preserving treatment by progestin]. Zhonghua Fu Chan Ke Za Zhi. 2013;48(7):519-522. Chinese.

19. Shan BE, Ren YL, Sun JM, et al. A prospective study of fertility-sparing treatment with megestrol acetate following hysteroscopic curettage for well-differentiated endometrioid carcinoma and atypical hyperplasia in young women. Arch Gynecol Obstet. 2013;288(5):1115-1123.

20. Zhou R, Yang Y, Lu Q, et al. Prognostic factors of oncological and reproductive outcomes in fertility-sparing treatment of complex atypical hyperplasia and low-grade endometrial cancer using oral progestin in Chinese patients. Gynecol Oncol. 2015;139(3):424-428.

21. LiYL. Treatment of 40 cases of early endometrial carcinoma with dydrogesterone combined with hysteroscopic resection and its effect on reproductive function (Chinese). Chin Foreign Med Res. 2017;15(7):102-103.

22. Yu M, Yang JX, Wu M, Lang JH, Huo Z, Shen K. Fertility-preserving treatment in young women with well-differentiated endometrial carcinoma and severe atypical hyperplasia of endometrium. Fertil Steril. 2009;92(6):2122-2124.

23. Qian H, Chen ZH. Clinical analysis of conservative treatment of 44 patients with early endometrial cancer (Chinese). Heilongjiang Med $J$ 2015;28(5):1111-1113.

24. Wang CJ, Chao A, Yang LY, et al. Fertility-preserving treatment in young women with endometrial adenocarcinoma: a long-term cohort study. Int J Gynecol Cancer. 2014;24(4):718-728.

25. Zhang Y. Therapeutic effect and pregnancy analysis of progesteronepreserving fertility in young patients with endometrial cancer and dysplasia (doctoral thesis). Tianjin Medical University; 2017. Chinese.

26. Yang YF, Liao YY, Liu XL, Su SG, Li LZ, Peng NF. Prognostic factors of regression and relapse of complex atypical hyperplasia and welldifferentiated endometrioid carcinoma with conservative treatment. Gynecol Oncol. 2015;139(3):419-423.

27. Yu M, Shen K, Yang JX. Outcome analysis of conservative treatment of well-differentiated endometrial adenocarcinoma and severe atypical hyperplasia in young women. Chin J Obstet Gynecol. 2006;41(4):242245. Chinese.

28. Koskas M, Uzan J, Luton D, Rouzier R, Daraï E. Prognostic factors of oncologic and reproductive outcomes in fertility-sparing management of endometrial atypical hyperplasia and adenocarcinoma: systematic review and meta-analysis. Fertil Steril. 2014;101(3):785-794.

29. Park JY, Kim DY, Kim JH, et al. Long-term oncologic outcomes after fertility-sparing management using oral progestin for young women with endometrial cancer (KGOG 2002). Eur J Cancer. 2013;49(4): 868-874.

30. Gallos ID, Yap J, Rajkhowa M, Luesley DM, Coomarasamy A, Gupta JK. Regression, relapse, and live birth rates with fertility-sparing therapy for endometrial cancer and atypical complex endometrial hyperplasia: a systematic review and metaanalysis. Am J Obstet Gynecol. 2012;207(4):266.e1-12.

31. Zhou H, Cao D, Yang J, Shen K, Lang J. Gonadotropin-releasing hormone agonist combined with a levonorgestrel-releasing intrauterine system or letrozole for fertility-preserving treatment of endometrial carcinoma and complex atypical hyperplasia in young women. Int $J$ Gynecol Cancer. 2017;27(6):1178-1182.

32. Kim MK, Yoon BS, Park H, et al. Conservative treatment with medroxyprogesterone acetate plus levonorgestrel intrauterine system for earlystage endometrial cancer in young women: pilot study. Int $J$ Gynecol Cancer. 2011;21(4):673-677.

33. Falcone F, Laurelli G, Losito S, di Napoli M, Granata V, Greggi S. Fertility preserving treatment with hysteroscopic resection followed by progestin therapy in young women with early endometrial cancer. $J$ Gynecol Oncol. 2017;28(1):e2. 
34. Giampaolino P, di Spiezio Sardo A, Mollo A, et al. Hysteroscopic endometrial focal resection followed by levonorgestrel intrauterine device insertion as a fertility-sparing treatment of atypical endometrial hyperplasia and early endometrial cancer: a retrospective study. J Minim Invasive Gynecol. 2018;S1553-S4650(18):30347-30349.

35. Li CM, Luo Y, Mao QX, Chen J. Analysis of 8 cases with early reproductive endometrial cancer treated by hysteroscopic resection combined with progesterone (Chinese). J Prac Obste Gynecol. 2013;29(3):224-226.

36. Wang Q, Guo Q, Gao S, et al. Fertility-conservation combined therapy with hysteroscopic resection and oral progesterone for local early stage endometrial carcinoma in young women. Int J Clin Exp Med. 2015;8(8):13804-13810.

37. Wang F, Yu A, Xu H, et al. Fertility preserved hysteroscopic approach for the treatment of stage Ia endometrioid carcinoma. Int $J$ Gynecol Cancer. 2017;27(9):1919-1925.

38. Covens A, Thomas G, Shaw P, et al. A phase II study of leuprolide in advanced/recurrent endometrial cancer. Gynecol Oncol. 1997;64(1): $126-129$.

39. Asbury RF, Brunetto VL, Lee RB, Reid G, Rocereto TF; Gynecologic Oncology Group. Goserelin acetate as treatment for recurrent endometrial carcinoma: a Gynecologic Oncology Group study. Am J Clin Oncol. 2002;25(6):557-560.

40. Gallos ID, Shehmar M, Thangaratinam S, Papapostolou TK, Coomarasamy A, Gupta JK. Oral progestogens vs levonorgestrel-releasing intrauterine system for endometrial hyperplasia: a systematic review and metaanalysis. Am J Obstet Gynecol. 2010;203(6):547.e1-10.

41. Yang B, Xie L, Zhang H, et al. Insulin resistance and overweight prolonged fertility-sparing treatment duration in endometrial atypical hyperplasia patients. J Gynecol Oncol. 2018;29(3):e35.

42. Minig L, Franchi D, Boveri S, Casadio C, Bocciolone L, Sideri M. Progestin intrauterine device and GnRH analogue for uterus-sparing treatment of endometrial precancers and well-differentiated early endometrial carcinoma in young women. Ann Oncol. 2011;22(3): 643-649.

43. Gadducci A, Spirito N, Baroni E, Tana R, Genazzani AR. The fertilitysparing treatment in patients with endometrial atypical hyperplasia and early endometrial cancer: a debated therapeutic option. Gynecol Endocrinol. 2009;25(10):683-691.

44. Park JY, Kim DY, Kim TJ, et al. Hormonal therapy for women with stage IA endometrial cancer of all grades. Obstet Gynecol. 2013;122(1):7-14.

45. Inoue $\mathrm{O}$, Hamatani $\mathrm{T}$, Susumu $\mathrm{N}$, et al. Factors affecting pregnancy outcomes in young women treated with fertility-preserving therapy for well-differentiated endometrial cancer or atypical endometrial hyperplasia. Reprod Biol Endocrinol. 2016;14:2.

46. Yu MJ, Liu QY. Hysteroscopic resection combined with progesterone for the treatment of early endometrial cancer with fertility preservation in 5 cases. Chin J Cancer Prev Treat. 2016; 23(s2):258-259. Chinese.

47. Deng ZW, Zhao YQ, Zeng X, Ye H, Qie MR. Outcomes and prognosis of early endometrial carcinoma in young patients with Hysteroscopy combined progesterone therapy (Chinese). J Pract Obstet Gynecol. 2017;5:366-369.

48. Hu CY, Tian YN, Ch L, Jia L, Liu TD. Effect of hysteroscopic electrotomy combined with progesterone on early reproductive endometrial cancer (Chinese). Clin Med China. 2016;32(4):332-334.

49. Chen R. Clinical analysis of 13 cases of fertility preservation of early endometrial cancer. Med Innovat China. 2015; 12(24):117119-117118. Chinese.

50. Tong XM, Lin XN, Jiang HF, Jiang LY, Zhang SY, Liang FB. Fertilitypreserving treatment and pregnancy outcomes in the early stage of endometrial carcinoma. Chin Med J (Engl). 2013;126(15):2965-2971.

51. Kalogera E, Dowdy SC, Bakkum-Gamez JN. Preserving fertility in young patients with endometrial cancer: current perspectives. Int $J$ Womens Health. 2014;6:691-701.
52. Ichinose M, Fujimoto A, Osuga Y, et al. The influence of infertility treatment on the prognosis of endometrial cancer and atypical complex endometrial hyperplasia. Int J Gynecol Cancer. 2013;23(2):288-293.

53. Chao AS, Chao A, Wang CJ, Lai CH, Wang HS. Obstetric outcomes of pregnancy after conservative treatment of endometrial cancer: case series and literature review. Taiwan J Obstet Gynecol. 2011;50(1):62-66.

54. Colombo N, Creutzberg C, Amant F, et al. ESMO-ESGO-ESTRO Consensus Conference on Endometrial Cancer: diagnosis, treatment and follow-up. Int J Gynecol Cancer. 2016;26(1):2-30.

55. Baek JS, Lee WH, Kang WD, Kim SM. Fertility-preserving treatment in complex atypical hyperplasia and early endometrial cancer in young women with oral progestin: is it effective? Obstet Gynecol Sci. 2016;59(1):24-31.

56. Gotlieb WH, Beiner ME, Shalmon B, et al. Outcome of fertility-sparing treatment with progestins in young patients with endometrial cancer. Obstet Gynecol. 2003;102(4):718-725.

57. Ramirez PT, Frumovitz M, Bodurka DC, Sun CC, Levenback C. Hormonal therapy for the management of grade 1 endometrial adenocarcinoma: a literature review. Gynecol Oncol. 2004;95(1): 133-138.

58. Ushijima K, Yahata H, Yoshikawa H, et al. Multicenter phase II study of fertility-sparing treatment with medroxyprogesterone acetate for endometrial carcinoma and atypical hyperplasia in young women. J Clin Oncol. 2007;25(19):2798-2803.

59. Perri T, Korach J, Gotlieb WH, et al. Prolonged conservative treatment of endometrial cancer patients: more than 1 pregnancy can be achieved. Int J Gynecol Cancer. 2011;21(1):72-78.

60. Park JY, Lee SH, Seong SJ, et al. Progestin re-treatment in patients with recurrent endometrial adenocarcinoma after successful fertilitysparing management using progestin. Gynecol Oncol. 2013;129(1): $7-11$.

61. Yamagami W, Susumu N, Makabe T, et al. Is repeated high-dose medroxyprogesterone acetate (MPA) therapy permissible for patients with early stage endometrial cancer or atypical endometrial hyperplasia who desire preserving fertility? J Gynecol Oncol. 2018;29(2):e21.

62. Tamauchi S, Kajiyama H, Utsumi F, et al. Efficacy of medroxyprogesterone acetate treatment and retreatment for atypical endometrial hyperplasia and endometrial cancer. J Obstet Gynaecol Res. 2018;44(1):151-156.

63. Yu M, Yang JX, Cao DY, Wang Y, Shen K. Diagnosis and management in patients with recurrent stage I endometrial cancer after primary fertility-preserving treatment (Chinese). J Shandong Univ. 2018;56(5): 23-29.

64. Lancaster JM, Powell CB, Kauff ND, et al. Society of Gynecologic Oncologists Education Committee statement on risk assessment for inherited gynecologic cancer predispositions. Gynecol Oncol. 2007;107(2):159-162.

65. Hoekstra AV, Kim JJ, Keh P, Schink JC. Absence of progesterone receptors in a failed case of fertility-sparing treatment in early endometrial cancer: a case report. J Reprod Med. 2008;53(11):869-873.

66. Hahn HS, Yoon SG, Hong JS, et al. Conservative treatment with progestin and pregnancy outcomes in endometrial cancer. Int J Gynecol Cancer. 2009;19(6):1068-1073.

67. Ai Z, Yin L, Zhou X, et al. Inhibition of survivin reduces cell proliferation and induces apoptosis in human endometrial cancer. Cancer. 2006;107(4):746-756.

68. Erkanli S, Kayaselcuk F, Kuscu E, et al. Expression of survivin, PTEN and p27 in normal, hyperplastic, and carcinomatous endometrium. Int J Gynecol Cancer. 2006;16(3):1412-1418.

69. Chen X, Zhang Z, Feng Y, et al. Aberrant survivin expression in endometrial hyperplasia: another mechanism of progestin resistance. Mod Pathol. 2009;22(5):699-708.

70. Gu C, Zhang Z, Yu Y, et al. Inhibiting the PI3K/Akt pathway reversed progestin resistance in endometrial cancer. Cancer Sci. 2011;102(3):557-564. 
71. Zhao S, Chen X, Lu X, Yu Y, Feng Y. Epidermal growth factor receptor signaling enhanced by long-term medroxyprogesterone acetate treatment in endometrial carcinoma. Gynecol Oncol. 2007;105(1):45-54.

72. Zhang Z, Dong L, Sui L, et al. Metformin reverses progestin resistance in endometrial cancer cells by downregulating GloI expression. Int $J$ Gynecol Cancer. 2011;21(2):213-221.
73. Ai Z, Wang J, Wang Y, Lu L, Tong J, Teng Y. Overexpressed epidermal growth factor receptor (EGFR)-induced progestin insensitivity in human endometrial carcinoma cells by the EGFR/mitogen-activated protein kinase signaling pathway. Cancer. 2010;116(15):3603-3613.

Cancer Management and Research

\section{Publish your work in this journal}

Cancer Management and Research is an international, peer-reviewed open access journal focusing on cancer research and the optimal use of preventative and integrated treatment interventions to achieve improved outcomes, enhanced survival and quality of life for the cancer patient. The manuscript management system is completely online and includes 